\title{
Validation of artificial defects for Non-destructive testing measurements on a reference structure
}

\author{
Maack Stefan ${ }^{1, *}$, Villalobos Salvador ${ }^{2}$ and Scott David ${ }^{2}$ \\ ${ }^{1}$ Bundesanstalt fuer Materialforschung und -pruefung (BAM), Non-Destructive Testing, Editorial Department, 12205 Berlin, \\ Germany \\ ${ }^{2}$ Electric Power Research Institute (EPRI), Advances Nuclear Technology (ANT), 28262 Charlotte, USA
}

\begin{abstract}
Non-destructive testing was established over the last decades as an important tool for assessing damages, material characterization and quality assurance in civil engineering. For example, Ground Penetrating Radar (GPR) can be used to scan large areas of concrete structures to determine the spatial position of the reinforcement. With the ultrasonic echo method, the thickness of concrete structures can be easily determined even if a high density of reinforcement is given. Various methods and processes have been developed for the validation of NDT procedures aiming at ensuring the quality of measurements in practical use. The Probability of Detection (POD) for example, is an available method to compare different technical devices with each other quantitatively regarding their performance. With this method, the best suited testing device for a specific inspection task under defined boundary conditions can be selected. By using the Guide to the Expression of Uncertainty in Measurement (GUM), it is possible to quantify the measurement uncertainty of an inspection procedure for a specific task. Another important aspect to improve the acceptance of Non-destructive testing methods is the development of reference specimens. Reference specimens serve for the calibration and further development of NDT methods under realistic conditions in different laboratories under the same conditions. A particular challenge here is the most realistic representation of a damage that can occur at building sites. Possible damages include for example horizontal and vertical cracks or honeycombs in concrete. Such a reference structure was built for the development of a new design of power plant constructions. Comparative studies on the manufacturing of realistic honeycombs and delaminations were carried out in advance on a test specimen. The results of this study are presented here.
\end{abstract}

\section{Introduction}

In recent decades, a variety of Non-destructive testing techniques have been developed for the assessment of concrete structures. The development of a new inspection technique often took place with regards to a specific question. For example, the eddy current method was developed for the exact determination of the concrete cover. Another measurement method that is linked to a specific test task is the half-cell potential test. This method is used to detect active corrosion areas in reinforced concrete. For these kind of measurement methods, developed for common inspection tasks, guidelines or standards often are provided. Examples of the documentation are available at national and international level [1-3].

In addition, there are serval measurement methods to solve a wide range of possible inspection tasks. Two of these methods are Ground Penetrating Radar (GPR) and Ultrasonic Pulse (USP). Due to the large spectrum of possible field inspection tasks, it is hardly possible to develop a generally applicable guideline or a standard.
The challenge for the user is to verify if the inspection task can be solved reliably with the chosen NDT method. This raises the question how to demonstrate the performance of the particular inspection method.

There are various possibilities to assess the performance of an NDT method. A qualitative estimation of the performance can be based on the experience of advanced users. Another possibility to quantify the performance is the use of Probability of Detection (POD). In [4,5] the statistical tool is used to detect reinforcement in concrete using GPR based in $[6,7]$. Another important point to assess the performance of NDT method is the determination of the measurement uncertainty. Corresponding with the state of the art, the uncertainty of measurement can be calculated according to the Guide to the Expression of Uncertainty in Measurements (GUM) [8]. When determining the uncertainty based on this guide, an uncertainty budget which considers the various boundary conditions of a measurement will be calculated. The advantage of this procedure is that the uncertainty budget can be extended

* Corresponding author: stefan.maack@bam.de 
depending on the different actual boundary conditions of the measurement on site [5].

To comprehensively assess a structure in civil engineering, it is often necessary to cover different inspection tasks with various Non-destructive testing methods. In [9] it is shown, how an assessment can be broken down to individual tasks and subtasks. Each of these sub-tasks must be assessed by a validated method [10]. To ensure the quality, inspection tasks must be clearly defined in cooperation with the client in advance. In [9-11], examples of such inspection tasks are described for reinforced concrete structures. In order to solve such a task successfully, it may be necessary to develop a reference specimen for the validation of NDT method.

This reference specimen must represent the task as realistically as possible. Once a reference specimen has been built, the performance of the NDT method can be demonstrated. There are no regulations for the development of reference specimens regarding the validation of Non-destructive testing methods in civil engineering yet. In [12], recommendations for the preparation of reference specimens in Nuclear industry are given.

The following article explains the procedure for producing such a reference structure. It shows how the task is defined and how in particular the validation of the reference test specimen takes place. At the beginning of the presentation, a brief introduction to the NDT method is presented.

\section{NDT technique and method}

\subsection{Pulse echo technique}

For these assessment, pulse echo technique is used. This kind of method has the advantage that only single-sided access to the component is required. In pulse echo mode, a short pulse (pulse: wave group containing serval frequencies) is coupled into the component. It will be partly reflected on objects and interfaces or penetrates them, while the transit time will be recorded by the receiving test probe on the accessible surface, Fig. 2. From the measured time, the spatial position of a reflector can be calculated by the ultrasonic velocity if the material is known. Fig. 2 shows a number of possible scenarios for pulse echo technique in schematic representation.

In pulse echo technique, the measured data are usually recorded along a line (B-scan) with a known offset of measuring points. The image of a single ultrasound signal in a time-of-flight diagram is called Ascan, Fig. 3 (left side). If the A-scans of a measuring line are lined up with colour-coded amplitude, a B-scan will be generated, Fig. 3 (middle). This B-scan corresponds to a projection (sectional image) through the examined structure. The color-coding of the A-scan is defined by the operator. In these examples a multi-color-coding (bright (low signal) - dark (high signal)) was chosen.

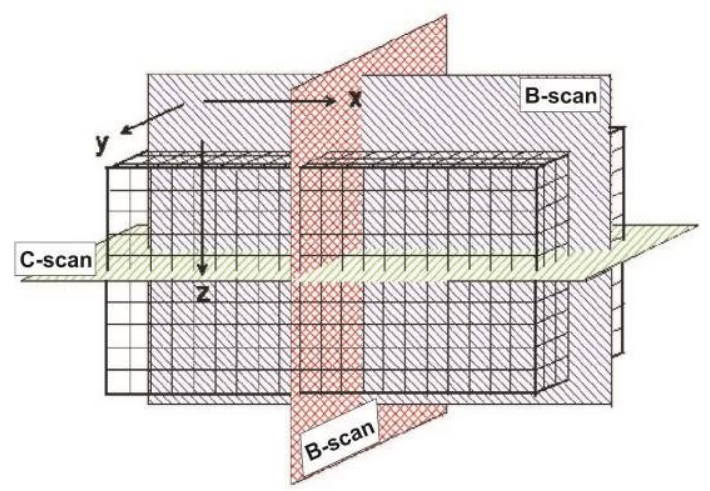

Fig. 1. Definition of B-scans and C-scan for volumetric NDT methods

If several B-scans are recorded in a grid with equivalent distances, a projection in a defined depth can then be calculated. This depth projection is called C-scan and gives a planar impression of the corresponding depth position, Fig. 1.

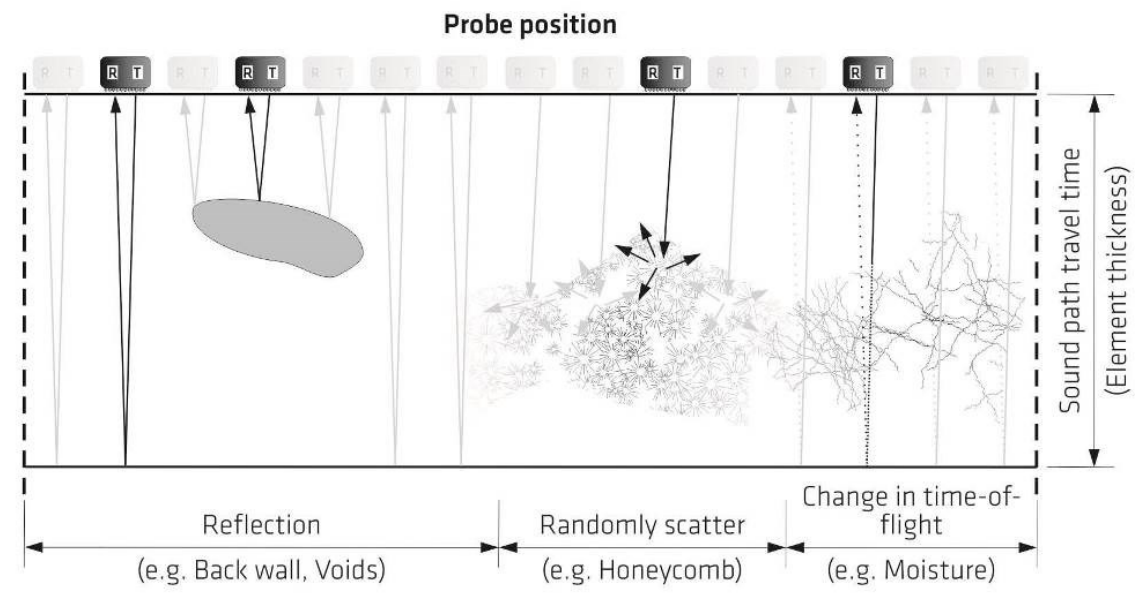

Fig. 2. Possible scenarios of pulse reflection for single-site measurements with volumetric NDT methods (from left to right: back wall-echo, reflection on void, randomly scatter e.g. on honey combs or compacting faults, change in time-of-flight e.g. micro cracking or moisture) 

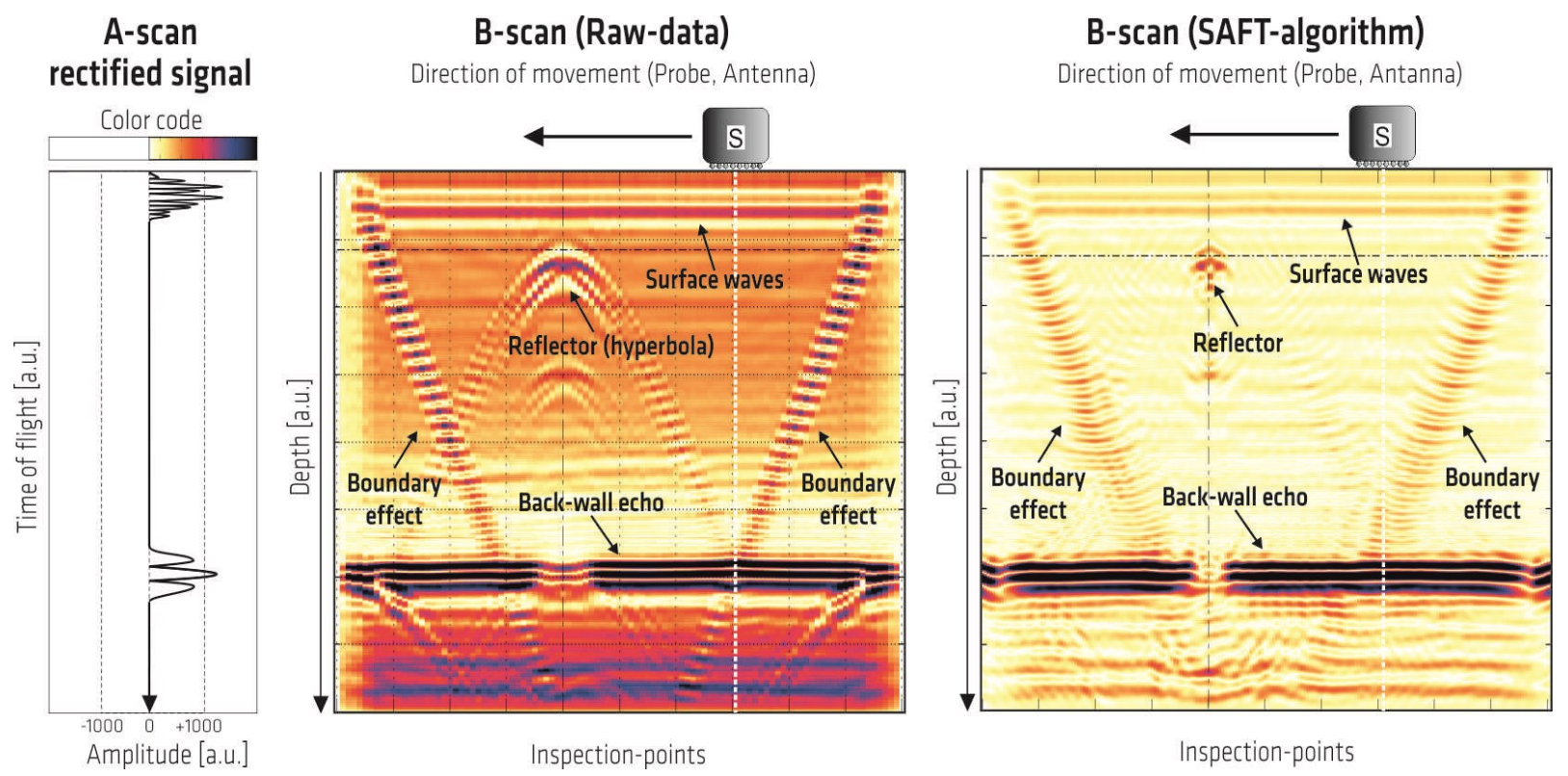

Fig. 3. Visualization of measurement data a processing results for an isotropic homogeneous material with single reflector (from left to right: A-scan (rectified signal), B-scan (raw-data), B-scan reconstructed data by SAFT-algorithm)

The probes currently used in civil engineering are characterized by a spatial directivity pattern wave field's radiation. With the help of reconstruction algorithms, this information can be converted into a spatially resolved signal image. As a reconstruction algorithm for the investigation carried out, the SAFT algorithm (SAFT - Synthetic Aperture Focusing Technique) [13-16] was used. As input parameter, the propagation velocity of pulse is required. In Fig. 3 on the right side the result of the reconstructed raw data is documented. The hyperbola of the single reflector is almost completely suppressed and the signal is focused on top of reflector.

\subsection{Ultrasonic (US)}

The ultrasonic pulse method can be used to solve a wide range of test tasks in different construction elements. This includes thickness measurements, the location estimation of structural elements or the integrity assessment of materials such as concrete.

Due to the heterogeneity of many materials, it is necessary to use a very low frequency range. This range is between $25 \mathrm{kHz}$ and $200 \mathrm{kHz}$. For typical concrete investigations transversal waves (SH-wave) are used. These waves are less sensitive to scattering effects of the aggregate materials. The velocity of the different types of waves depends on the examined materials. Typical for ultrasonic waves in concrete are about $4.000 \mathrm{~m} / \mathrm{s}$ for longitudinal and $2.700 \mathrm{~m} / \mathrm{s}$ for transversal waves, which results in wavelengths in the range of $2 \mathrm{~cm}$ to $8 \mathrm{~cm}$.

In Fig. 4., the typical ultrasonic equipment consisting of a probe, a handheld device and a computer for evaluation of the recorded data can be seen. For the coupling of the probe no coupling agent is required. The excitation takes place here by probes that operate on the piezoelectric principle. Transmitter and receiver are separated to avoid disturbing effects.

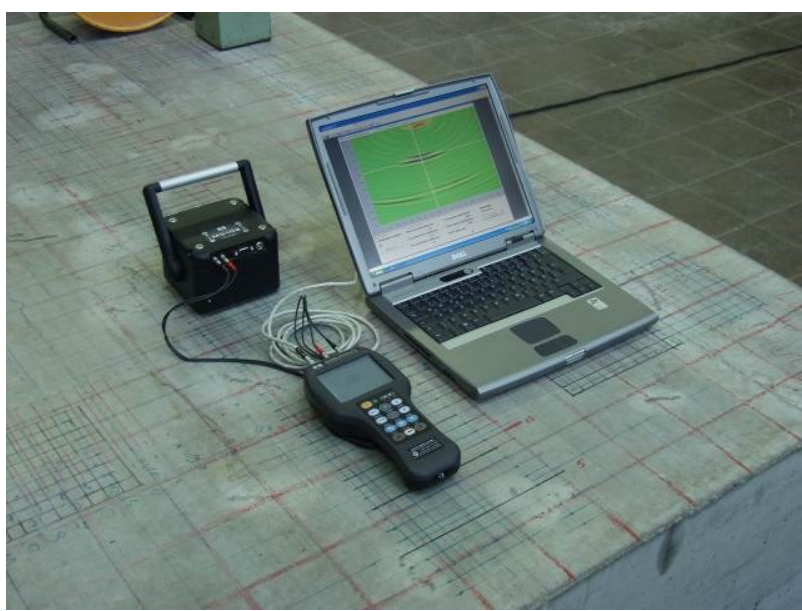

Fig. 4. Standard Ultrasonic equipment for concrete inspection consisting of three basic components (handheld, probe, computer for data analysis)

\section{Reference structure}

The reference structure is a Mockup of a new design used in power plant constructions. This consists of two opposite steel plates which are prefabricated. The two steel plates are firmly connected by constructive elements. Between the two steel plates, the construction is filled with concrete. This backfill of concrete has to be inspected for integrity. For this purpose, the construction should be tested with Non-destructive testing methods. In this context, the question has to be answered, if honeycombs or delamination's are present. The reference structure can only be tested from the sides where the steel plates are located. Fig. 5 schematically shows the reference structure in a sectional view, where one steel plate and connecting elements are displayed. Green spheres and squares visualize the location of possible defects. 


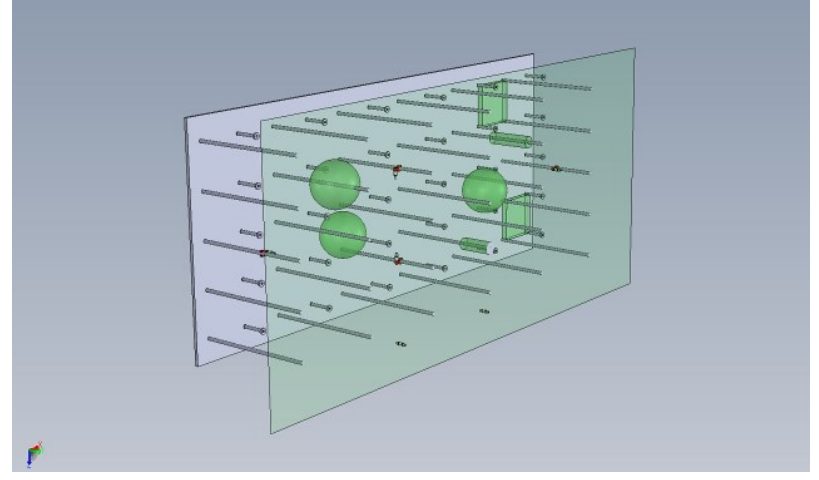

Fig. 5. Principle design of reference specimen (Mockup) indicate possible defects (honeycombs, delamination's) with conceivable positioning of artificial defects e.g. honeycombs or delamination's

The reference structure will be used to compare different Non-destructive testing methods with regards to determining the location of the artificial defects. The service provider only receives the information about the size and the kind of defect. Furthermore, the service provider is informed, which areas are without defects. The challenge is to analyse these possible damage scenarios as realistically as possible. For this purpose, another test specimen will be built in advance. In this specimen different types of artificial honeycombs are integrated as well as a design of artificial delamination.

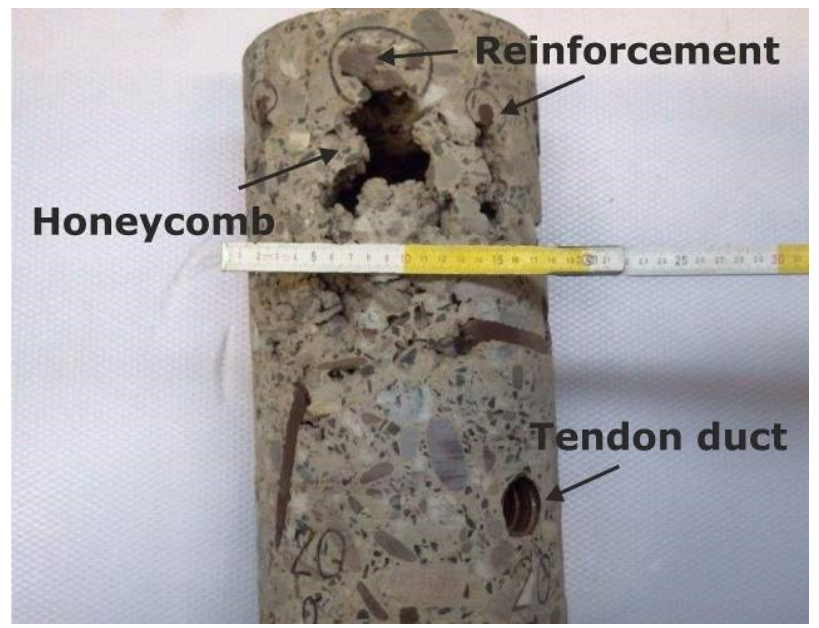

Fig. 6. Drilling core from real structure with honeycomb/ void under reinforcement bars

Fig. 6 shows a drill core that was taken as a part of the investigations on a bridge deck. It can be seen that honeycombs of various sizes have formed below the reinforcement bars. The picture shows very clearly that the surface of the honeycomb has an irregular surface. This is typical for this kind of defects.

\section{Test specimen}

In [9] a honeycomb is defined as: '(...) irregular volume in concrete with significantly reduced density. The size of the voids are on the order of the aggregate size (...)' Frequently artificial honeycombs are simulated using foam material. However, this does not present a realistic segregation of the concrete. While defects made of foam have comparatively plane surfaces, a realistic honeycomb has an irregular surface, Fig. 6. Thus, the result is a diffuse scattering reflection of the incident ultrasonic pulse. In the context of a preliminary experiment, the difference between these two damage characteristics of artificial honeycombs should be shown.

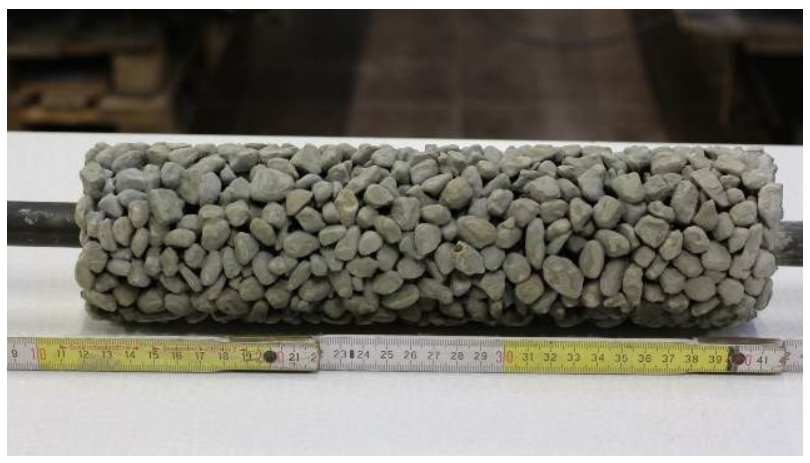

Fig. 7. Artificial honeycomb on reinforcement bar without finished coating during production process

In Fig. 7, the developed artificial honeycomb along a rebar can be seen. It consists of an agglomeration of stones with a constant diameter of $1.6 \mathrm{~cm}$. This diameter corresponds with the largest aggregate of the concrete mixture used. The honeycomb has a total length of $30 \mathrm{~cm}$ and a diameter of $9 \mathrm{~cm}$. The single aggregates are glued using a cement paste. To avoid the cement paste flowing into the voids during pouring, the honeycomb is coated with a thin layer of cement paste. The prepared honeycomb has a bulk density of $0.75 \mathrm{~g} / \mathrm{cm}^{3}$. For comparison, an artificial honeycomb made of foam was produced as well. This defect has a bulk density of $0.02 \mathrm{~g} / \mathrm{cm}^{3}$. The geometric dimensions are similar to the other. The final position can be seen in Fig. 8. They have the same concrete cover of $9.5 \mathrm{~cm}$.

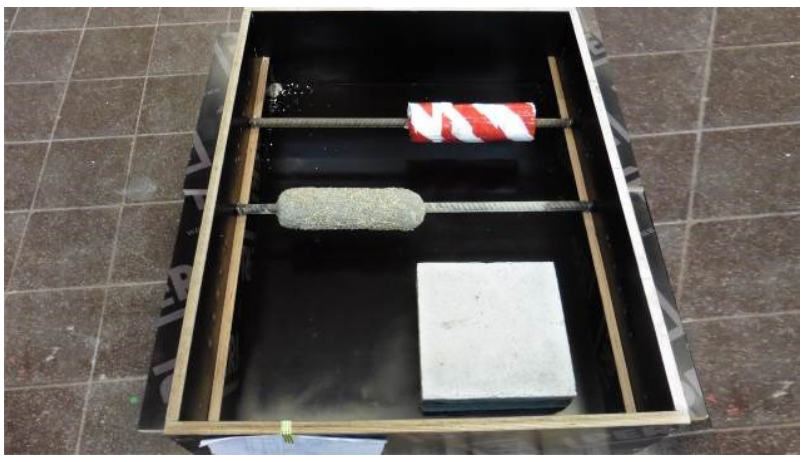

Fig. 8. Prepared test specimen with different artificial defects before pouring concrete

In addition to the comparison of the two types of honeycombs, an artificial delamination is placed in the specimen as well. A delamination is defined in [9] as: (...) locally debonding in concrete (...) parallel to the surface. '. Typically, it is filled with air. In the past, trials have often been made to simulate delaminations by attaching thin foils or by applying oil on metal surfaces. In the context of this experiment, it will be shown that a 
delamination can also be produced by using two thin concrete slabs.

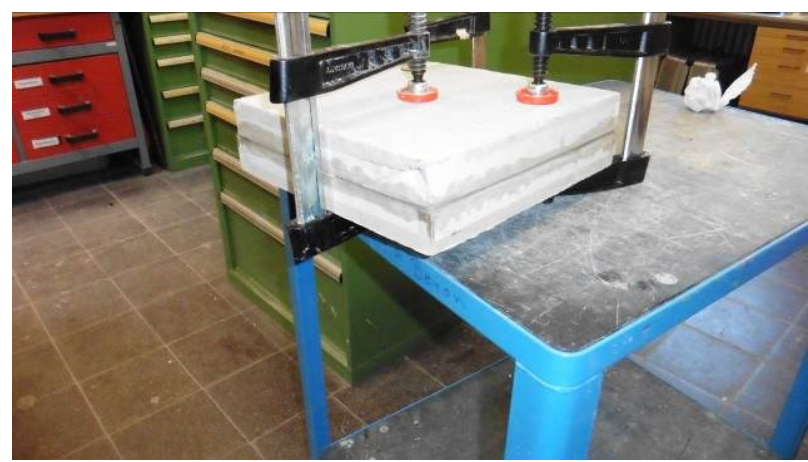

Fig. 9. Two fixed concrete plates with a small air gap between each other to simulate a delamination

Fig. 9 shows the making of the artificial delamination. Here, two concrete slabs, each with a thickness of $4 \mathrm{~cm}$, were put together. To ensure the position of the concrete slabs they were fixed at the sides with a thin joint of silicone. The square concrete slabs have an edge length of $30 \mathrm{~cm}$ and were made from the same concrete mixture as the test specimen. This is the prerequisite for getting the lowest possible acoustic contrast on this interface (Fig. 11, Detail 01 - red filled triangles).

\section{Validation}

The artificial defects are measured destructively to validate the size of the defects. For this purpose, the test specimen is sawn into several elements. In order to stabilize the voids, they are filled with a synthetic resin.
This filling of the defects can be seen in Fig. 10. The resin has two tasks here. First, it stabilizes the voids during the sawing process. Secondly, the synthetic resin is a material that fluoresces under UV light. Thus, after the test specimen has been cut, it is possible to detect the filled voids easily.

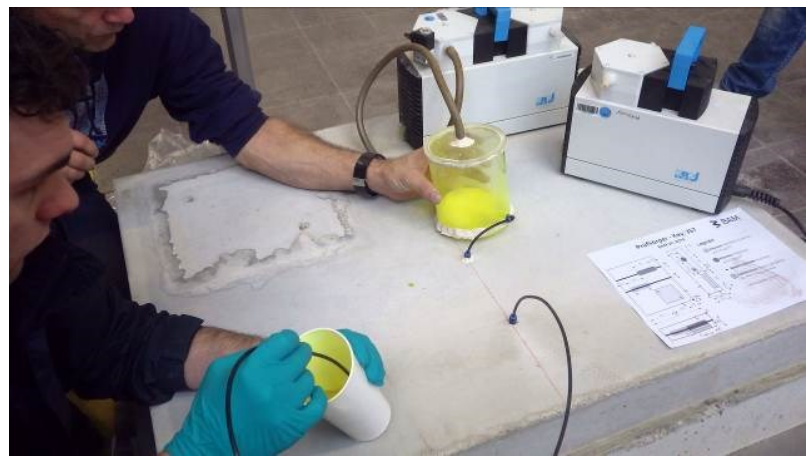

Fig. 10. Filling the air voids with fluorescent resin using a vacuum pump

After validating the geometrical position of the various components, the visual inspection was carried out with the UV light. In Fig. 11 (left side) in a depth of $30 \mathrm{~cm}$ the honeycomb made of foam is located. In the middle of it, the rebar can be seen. On the right side of the figure the artificial delamination is located. In the detail image (Detail 02), it can be seen very clearly under UV light that the delamination did not fill with cement during pouring and can now be measured having about $0.3 \mathrm{~mm}$. In detail image (Detail 01) the interface between the concrete slab and the concrete can be seen. This is highlighted by three red arrows. It shows very clearly that no boundary layer can be seen here.

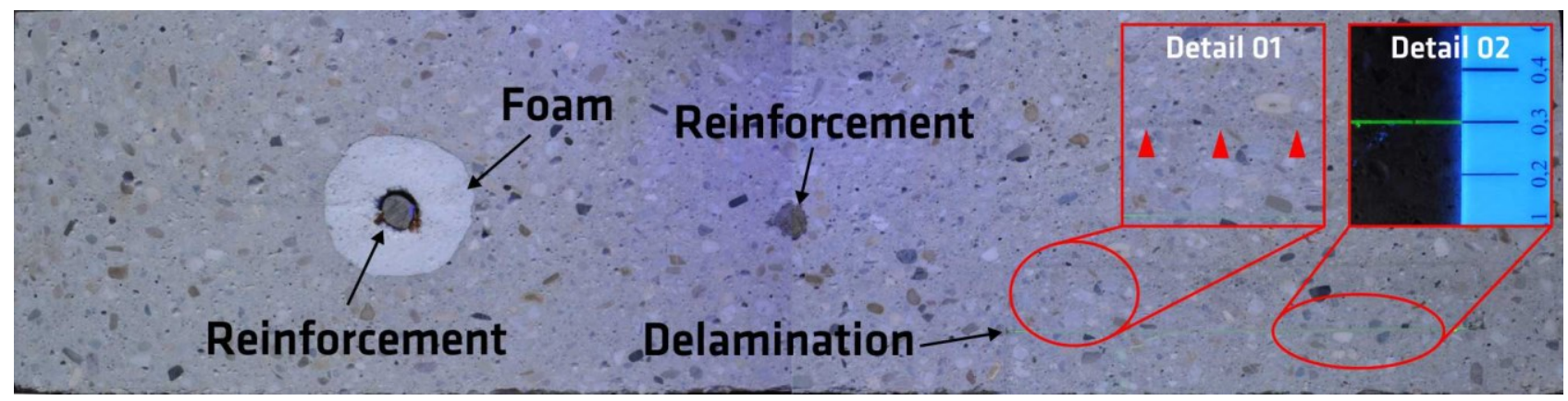

Fig. 11. Concrete slice at $30 \mathrm{~cm}$ with artificial honeycomb made of foam (left side) and delamination filled with fluorescent resin illuminated by ultraviolet light (right side)

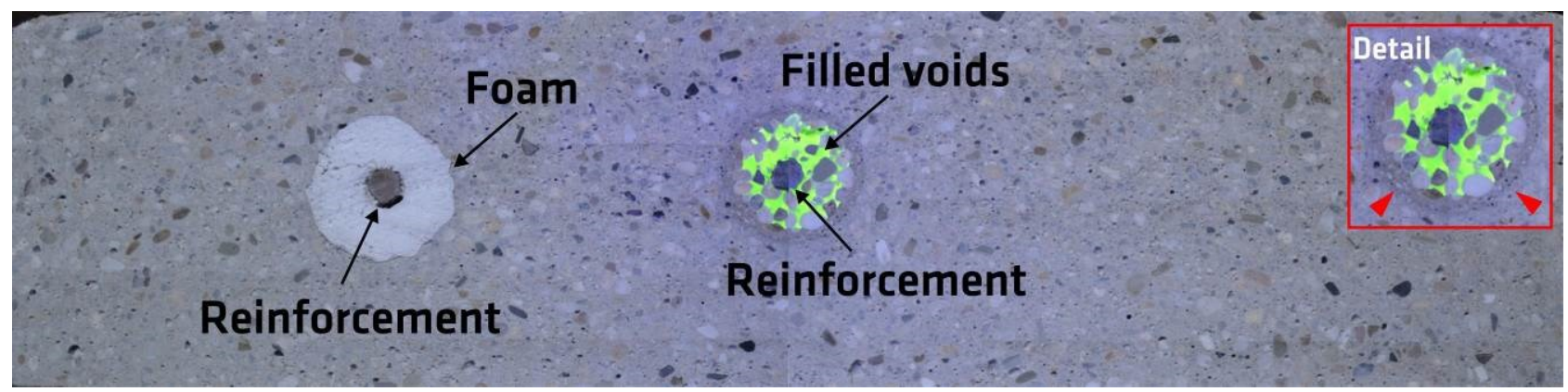

Fig. 12. Concrete slice at $48 \mathrm{~cm}$ with artificial honeycomb made of foam (left side) and artificial honeycomb made of real aggregate illuminated by ultraviolet light (right side) 
Fig. 12 shows the cut at $48 \mathrm{~cm}$. Both honeycombs can be seen, on the left side the honeycomb consisting of foam on the right side the one made of the real aggregate. The voids of the honeycomb on the right are completely filled with the synthetic resin. Under UV light, these areas glow in a yellowish hue. That shows that no cement paste has penetrated into the voids during the casting process (Detail). The irregular surface corresponds to that of a real honeycomb (Fig. 6, drill core).

Artificial honeycombs can be made by using real aggregates and cement paste. During pouring, the voids are preserved. By stacking two thin concrete slabs, an artificial delamination can be created and the void in between is stable. Since the concrete slabs are made with the same recipe, the interface to the surrounding concrete of the test specimen is difficult to detect visually.

\section{US Results}

The following section presents the analysed results of the NDT measurements. The tests were carried out 12 weeks after the preparation of the test specimen. In order to enable a precise measurement, an automated scanner system is used [17]. The size of the measured area is $70 \mathrm{~cm}$ in the y-direction and $88 \mathrm{~cm}$ in the $\mathrm{x}$-direction with a distance of $1 \mathrm{~cm}$. In sum 6.319 measurement points were recorded.

All measurements were carried out with transverse waves and a mid-frequency of $50 \mathrm{kHz}$. The waves are polarized in the y-direction, the calibrated velocity is $2.450 \mathrm{~m} / \mathrm{s}$. Based on this calibrated ultrasonic velocity, all measurement data were reconstructed with SAFT algorithm.

All results are presented in C-scans in selected depths, with the same color code. The dataset is also normalized to the maximum amplitude. A dark color represents a high signal strength, while a light color means a low signal strength. In the respective $\mathrm{C}$-scans the location of the artificial honeycombs and of the delamination are indicated by gray lines based on the validation. The depth of the $\mathrm{C}$-scan is marked by a dashed red line in the right-hand section.

Fig. 13 shows the C-scan at a depth of $10.3 \mathrm{~cm}$. This corresponds approximately to the upper edge of the artificial honeycombs in a concrete cover of about $9.5 \mathrm{~cm}$. The upper honeycomb is made of foam. It is very easy to see that a clear linear reflection in the size of the defect is reflected. In the area of the realistic honeycomb, no reflection can be seen at this depth. The reason is the desired diffusive scattering of ultrasonic waves at the irregular surface (Fig. 12, Detail) of the defect. In contrast, at the interface from concrete to foam with its relatively smooth surface (Fig. 11) a very strong reflection appears. The deviation in the depth is due to the geometry of the honeycomb. Due to the curved surface, the SAFT algorithm does not focus optimally, which leads to an apparent change at the reconstructed depth.

Fig. 14 shows the C-scan at a depth of $25 \mathrm{~cm}$, where the back-wall reflection is expected. The back-wall of the test specimen is here characterized by a dark colour manifested by a high signal strength. In some areas of the C-scan, it can be seen that the back-wall echo is missing. In the upper area, the shading by the honeycombs in the lower area is due to the delamination. It can be seen very well that both honeycombs can be indirectly detected by the absence of the back-wall echo. Areas where the back-wall echo is missing, correspond very well with the validated position of the defects. Furthermore, the reinforcement bars are also indicated by the absence of the back-wall echo.
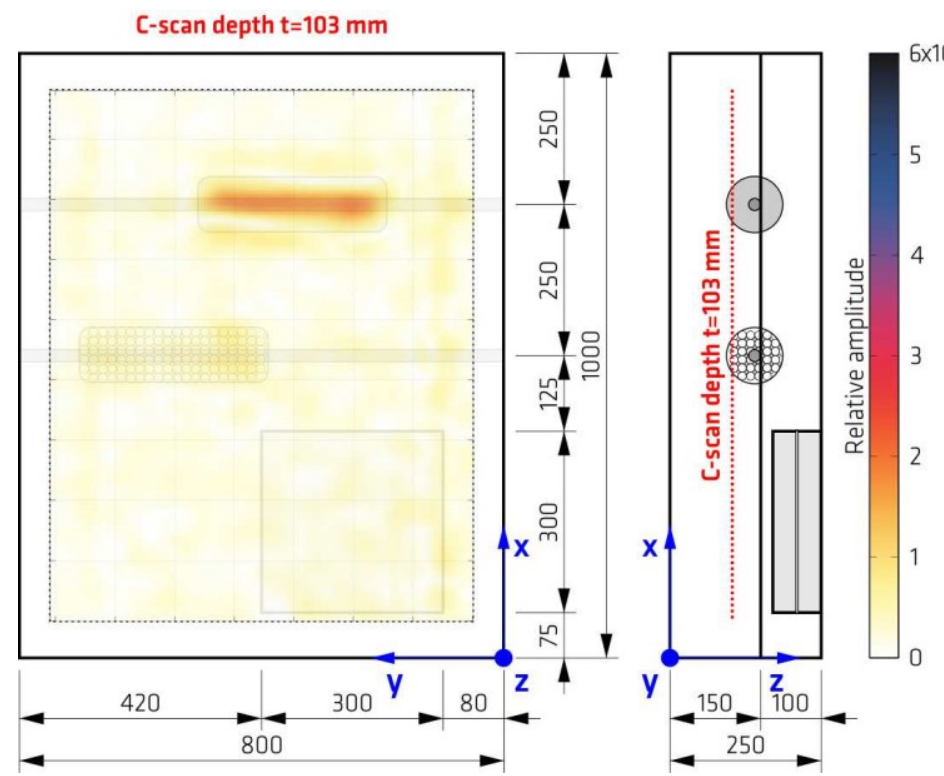

Legend:

Honeycomb: Aggregate size $D=16 \mathrm{~mm}$; $\rho=0,75 \mathrm{~g} / \mathrm{cm}^{3}$ (Bulk density)

Honeycomb: Foam; $\rho=0,02 \mathrm{~g} / \mathrm{cm}^{3}$ (Bulk density)

- Reinforcement: $D=20 \mathrm{~mm}$

Delamination: Concrete-Air-Concrete; $40 \mathrm{~mm}$ - $0 \mathrm{~mm}-40 \mathrm{~mm}$

\section{All geometric data in $\mathrm{mm}$}

Fig. 13. C-scan of Ultrasonic measurement in depth of $10.3 \mathrm{~cm}$ with a linear reflector at the position of an artificial honeycomb made of foam (red dashed line illustrates depth of C-scan in view of y-direction) 

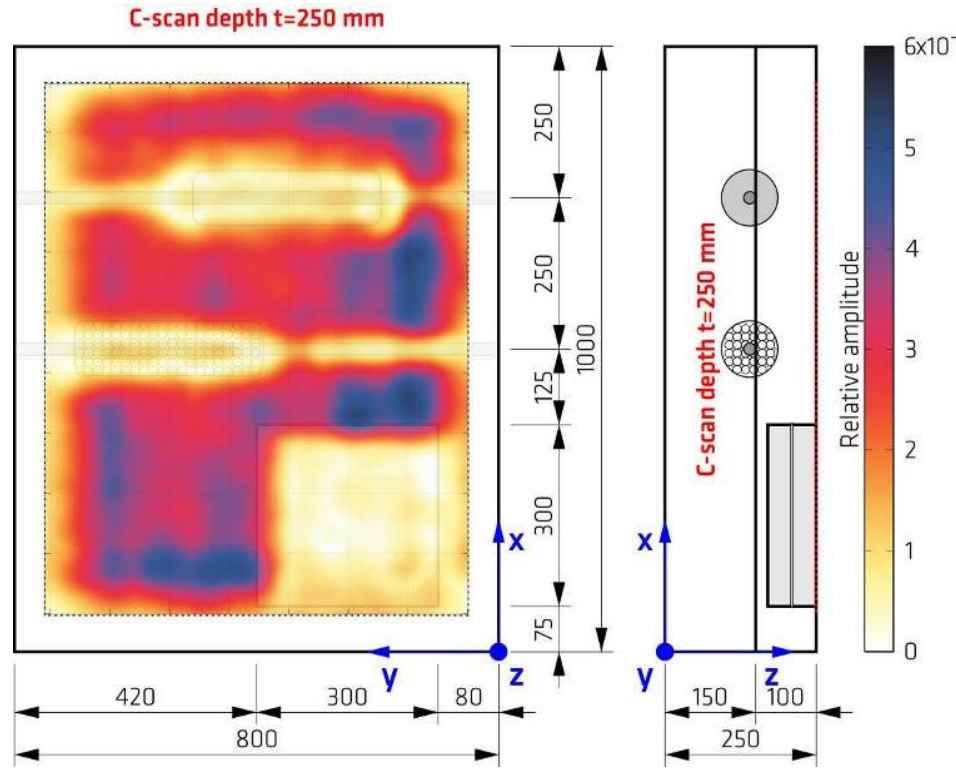

\section{Legend:}

Honeycomb: Aggregate size $D=16 \mathrm{~mm}$; $\rho=0,75 \mathrm{~g} / \mathrm{cm}^{3}$ (Bulk density)

Honeycomb: Foam; $\rho=0,02 \mathrm{~g} / \mathrm{cm}^{3}$ (Bulk density)

- Reinforcement: $D=20 \mathrm{~mm}$

\section{Delamination: Concrete-Air-Concrete; 40 mm - 0 mm - 40 mm}

\section{All geometric data in $\mathrm{mm}$}

Fig. 14. C-scan of Ultrasonic measurement in depth of $25 \mathrm{~cm}$ with shadowed back wall signal at positions of the artificial honeycomb made of foam, made of real aggregate and the delamination (red dashed line illustrates depth of C-scan in view of y-direction)

Fig. 15 shows the $\mathrm{C}$-scan at a depth of $17 \mathrm{~cm}$. This corresponds to the top edge of the thin concrete slab of the artificial delamination. In this $\mathrm{C}$-scan various weak reflections can be seen. On one hand, it shows single linear reflectors of the two reinforcing bars, on the other hand, it indicates a flat reflector in the area of the delamination. The reflections of the rebars are signal components whose maximum is located at a depth of about $14 \mathrm{~cm}$. This is the depth, where the rebars were installed. The weak reflection in the area of delamination is the result of the two concrete layers, which were manufactured at different times. This shows that the

\begin{abstract}
ultrasonic echo method is very sensitive to such interfaces.
\end{abstract}

Fig. 16 shows the $\mathrm{C}$-scan at a depth of $21 \mathrm{~cm}$. This is the depth at which the air layer of the artificial delamination is located (Fig. 11, Detail 02). It can be seen that a very strong reflection is emerging here, where the reflector corresponds to the geometry of the artificial delamination. As expected, an air-filled delamination leads to a total reflection of the transverse wave. This implies that the ultrasound method provides no additional information from below the delamination. This is also confirmed in Fig. 14 by shading the backwall echo in this area.
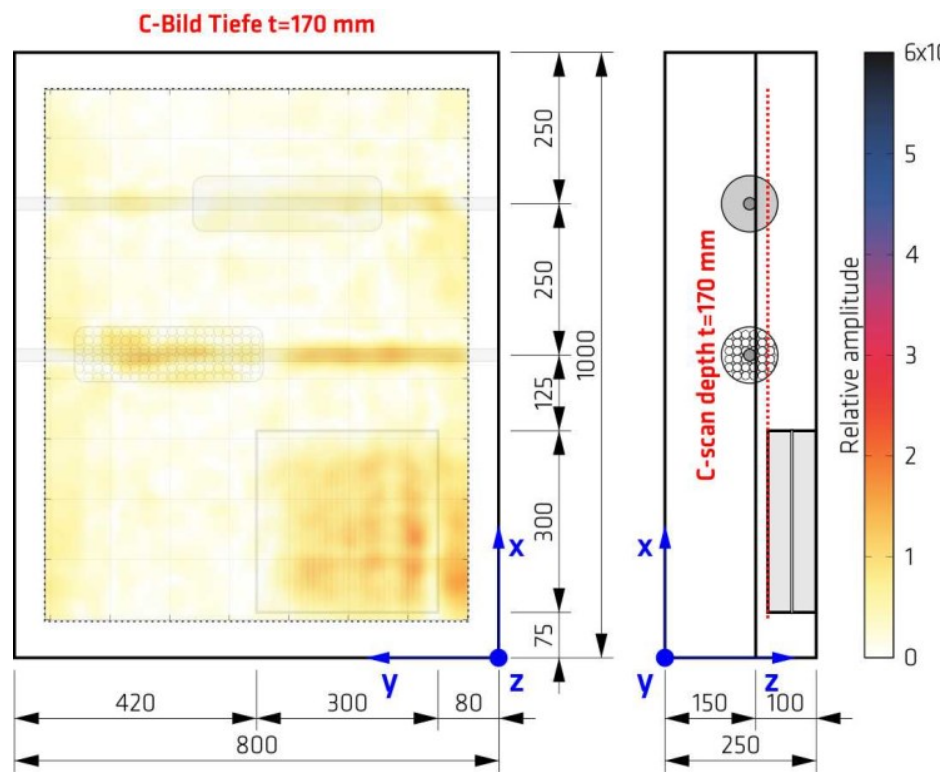

\section{Legend:}

Honeycomb: Aggregate size $D=16 \mathrm{~mm}$; $\rho=0,75 \mathrm{~g} / \mathrm{cm}^{3}$ (Bulk density)

Honeycomb: Foam; $\rho=0,02 \mathrm{~g} / \mathrm{cm}^{3}$ (Bulk density)

- Reinforcement: $D=20 \mathrm{~mm}$

Delamination: Concrete-Air-Concrete; 40 mm - 0 mm - 40 mm

All geometric data in $\mathrm{mm}$

Fig. 15. C-scan of Ultrasonic measurement in depth of $17 \mathrm{~cm}$ with slight linear reflectors at the positions of reinforcement bars and in the area of the delamination (red dashed line illustrates depth of C-scan in view of y-direction) 

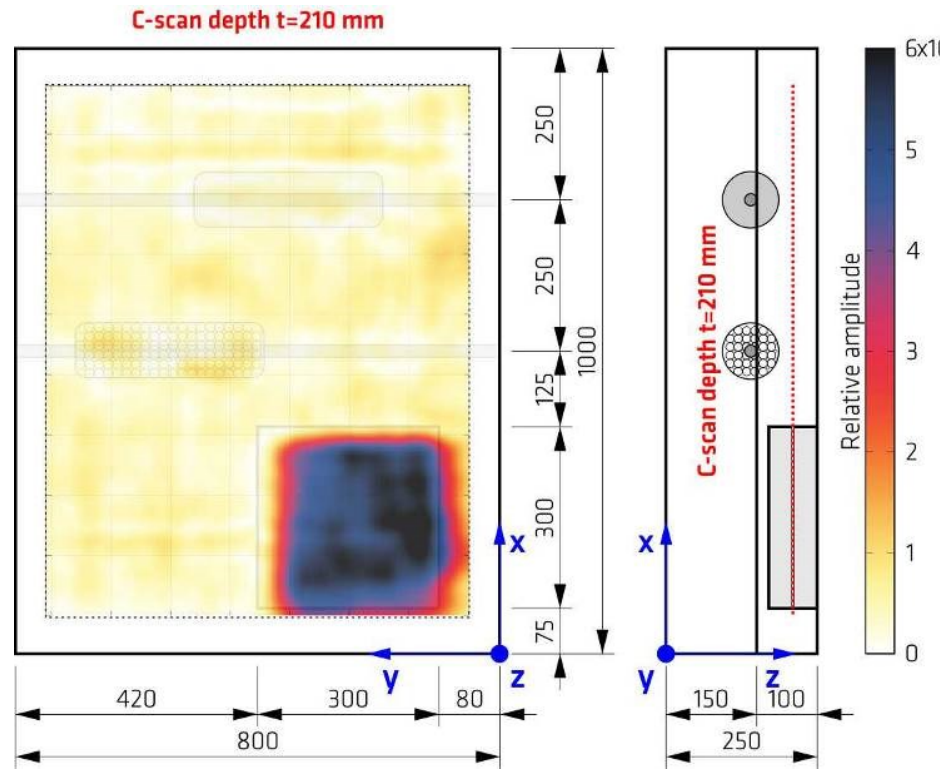

Legend:

Honeycomb: Aggregate size $D=16 \mathrm{~mm}$; $\rho=0,75 \mathrm{~g} / \mathrm{cm}^{3}$ (Bulk density)

Honeycomb: Foam; $\rho=0,02 \mathrm{~g} / \mathrm{cm}^{3}$ (Bulk density)

○ Reinforcement: $D=20 \mathrm{~mm}$

Delamination: Concrete-Air-Concrete; $40 \mathrm{~mm}$ - $0 \mathrm{~mm}-40 \mathrm{~mm}$

All geometric data in $\mathrm{mm}$

Fig. 16. C-scan of Ultrasonic measurement in depth of $21 \mathrm{~cm}$ with strong reflector in the area of the delamination (red dashed line illustrates depth of C-scan in view of y-direction)

\section{Conclusion}

During the planning and making of a reference specimen, it should be tested how realistic artificial defects can be produced in advance. The aim of the reference specimen is to demonstrate the integrity of the design using Non-destructive testing methods. For this purpose, possible damage scenarios, which can occur during the manufacturing process of the real structure or in particular during the concrete pouring, are defined. The classic damage scenarios in this case are honeycombs or delaminations. In the past, artificial honeycombs were often simulated by introducing foam pieces into the concrete. In the context of these investigations, it should be shown how realistic this type of production of the honeycombs is. In addition, it was investigated how a suitable artificial delamination can be produced.

In the first step, a test specimen was produced, which contains two different variants of honeycombs. Their properties were tested. Furthermore, an artificial delamination was installed. In the second step, investigations were carried out with the ultrasonic echo method. These results were compared and evaluated. The validation of the result was carried out by destructively examining the test specimen.

It was shown that honeycombs made of foam material are not always suitable for realistic simulation of segregation phenomena in concrete. Such production can lead to possible misinterpretations. The detection of the artificial honeycomb consisting of real aggregates is much more difficult in comparison. This is due to the diffusive scattering of elastic waves, which corresponds more to real field defects.

In addition to these investigations, the possibility of producing a delamination was investigated. It was shown that with two concrete slabs attached to each other, a thin air gap of less than one millimetre can be created. The
Non-destructive tests show that this type of artificial delamination can be produced without the introduction of foreign materials such as foils.

In conclusion, it is recommended to carry out a detailed analysis of the possible damage scenarios for imaging. Furthermore, preliminary tests should be carried out to check how realistic the artificial defects are. With this procedure, misinterpretations on the real components can be avoided. Thus, as a result, the performance of Non-destructive testing can be significantly improved.

\section{References}

1. Forde, M. C.: Report on nondestructive test methods for evaluation of concrete in structures: Reported by ACI Committee 228. ACI 228.2R-13. American Concrete Institute, Farmington Hills, Mich., (2013).

2. Rupnow, T. D.; Icenogle, P.: Evaluation of Surface Resistivity Measurements as an Alternative to the Rapid Chloride Permeability Test for Quality Assurance and Acceptance: Technical Report. FHWA/LA.11/479, Baton Rouge, (2011).

3. Deutsches Institut für Normung e.V.: Testing concrete in structures - Part 2: Non-destructive testing - Determination of rebound number; English version EN 12504-2:2012, ICS 91.100.30. Beuth Verlag, Berlin, (2012).

4. Feistkorn, S.: Rebar detection - POD approach to determine the reliability of GPR systems and to quantify the influence of different material parameters. The proceedings of 2016 16th International Conference on Ground Penetrating Radar (GPR): 13-16 June 2016, The Hong Kong Polytechnic University, Department of Land Surveying and Geo-Informatics. IEEE, Piscataway, NJ, 2016, S. 1-6 (2016). 
5. Feistkorn, S.; Taffe, A.: Methods to Assess the Quality of Non-Destructive Testing in Civil Engineering Using POD and GUM for Static Calculations of Existing Structures*. Materials Testing 56, 7-8, S. 611-616 (2014).

6. Annis, C.: NONDESTRUCTIVE EVALUATION SYSTEM RELIABILITY ASSESSMENT: DEPARTMENT OF DEFENSE Handbook. MILHDBK-1823-2, (2004).

7. Berens, A. P.: NDE Reliability Data Analysis. In: Davis, J. (Hrsg.): ASM METALS HANDBOOK: Nondestructive Evaluation and Quality Control. VOLUME 17. ASM International, 1437-1470 (1989).

8. JCGM: Evaluation of measurement data - Guide to the expression of uncertainty in measurement 17.020. Joint Committee for Guides in Metrology (2008).

9. Wiggenhauser, H.; Azari, H.: Classification of Nondestructive Evaluation Tasks for Reinforced Concrete Structures. Journal of Infrastructure Systems 23, 4, S. 4017021 (2017).

10. Taffe, A.; Gehlen, C.: Methodology for the validation of NDT-CE methods using transit time measurement. In: NDT.net (Hrsg.). 7th International Symposium on Nondestructive Testing in Civil Engineering (2009).

11. Hoła, J.; Bień, J.; Sadowski, Ł.; Schabowicz, K.: Non-destructive and semi-destructive diagnostics of concrete structures in assessment of their durability. Bulletin of the Polish Academy of Sciences Technical Sciences Vol. 63, No. 1, 87-96 (2015).
12. Gandossi, L.; Atkinson, I.; Booler, R.; Bouvret, Y.; Chapman, R.; Daniels, W.; Dawood, T.; Horacek, L.; Horvath, M.; Jardet, P.; Jonsson, A.; Kelsey, P.; Kuusinen, P.; Martin, E.; Martinsen, H.; Moussebois, D.; Neundorf, B.; Schwammberger, R.; Martin, O.; Söderstrand, H.; Pitkänen, J.: ENIQ recommended practice 5: Guidelines for the design of test pieces and conduct of test piece trials. ENIQ report No 42. Publications Office, Luxembourg, (2011).

13. Seydel, J.: Ultrasonic synthetic-aperture focusing techniques in NDT. In: ed. By R.S. Sharpe (Hrsg.): Research Techniques in Nondestructive Testing. Academic Press, London, S. pp. 1-47 (1982).

14. Schickert, M.; Krause, M.; Müller, W.: Ultrasonic Imaging of Concrete Elements Using Reconstruction by Synthetic Aperture Focusing Technique. Journal of Materials in Civil Engineering 15, 3, S. 235 (2003).

15. Langenberg, K.; Fellinger, P.; Marklein, R.; Zanger, P.; Mayer, K.; Kreutter, T.: Inverse Methods and Imaging. In: Achenbach, J. D. (Hrsg.): The Evaluation of materials and structures by quantitative ultrasonics. Springer Verlag, Wien New York, S. 318-398 (1993).

16. Langenberg, K.-J.; Marklein, R.; Mayer, K.: Ultrasonic nondestructive testing of materials: Theoretical foundations. CRC Press, Boca Raton, FL (2012)

17. Wiggenhauser, H. : Automated NDE of structures with combined methods. Nondestructive testing of materials and structures, Springer, Netherlands, 753-760 (2013). 